\title{
NON-RESONANT TUNNELLING IN SHORT-PERIOD SUPERLATTICES WITH OPTICAL CAVITIES
}

\author{
M.S. Kagan a, I.V. Altukhov ${ }^{\text {a }}$, S.K. Paprotskiy a , A.N. Baranov ${ }^{\text {b }}$, R. Teissier ${ }^{\text {b }}$, \\ N.D. Il'inskaya ${ }^{c}$, A.A. Usikova ${ }^{c}$, A.D. Buravlev ${ }^{c}$, and V.M. Ustinov ${ }^{c}$ \\ ${ }^{a}$ Kotelnikov Institute of Radio Engineering and Electronics, Russian Academy of Sciences, Mokhovaya 11-7, \\ 125009 Moscow, Russia \\ E-mail: kagan@cplire.ru \\ ${ }^{\mathrm{b}}$ IES, Université Montpellier 2, CNRS, 34095 Montpellier Cedex 5, France \\ 'Ioffe Physical Technical Institute, Russian Academy of Sciences, Politekhnicheskaya 26, 194021 St. Petersburg, Russia
}

Received 18 November 2013; accepted 4 December 2013

\begin{abstract}
The vertical transport in short-period InAs/AlSb and GaAs/AlAs superlattices was studied. The negative differential conductivity was observed in a miniband transport regime as a result of the overlapping of confined states in a periodic quantum well structure (Esaki-Tsu mechanism). Several maxima appeared on current-voltage characteristics of the superlattices found in the non-resonant tunnelling regime. They are shown to be due to the influence of the optical cavity on optical transitions within quantum wells (Purcell effect).
\end{abstract}

Keywords: superlattices, non-resonant tunnelling, optical cavities, Purcell effect

PACS: 73.21.Cd, 73.40.Gk, 73.61.Ey, 78.67.Pt

\section{Introduction}

In recent years, much attention has been focused on studies of InAs-based type II heterostructures due to the large conduction band offset and small electron effective mass $\left(0.023 m_{0}\right.$ in InAs). The studies have been performed mainly in InAs/AlSb structures aimed for mid-IR (see, e. g. [1-3] and references therein) and $\mathrm{THz}$ [4] photodetectors, quantum cascade lasers operating in the spectral range of 2.3 to $5 \mu \mathrm{m}$ (e. g. [5-7]) and fast hot-electron transistors [8]. Transport properties of superlattices (SLs) (mainly GaAs-based SLs) have been studied in detail because of expected Bloch wave amplification, which is promising for $\mathrm{THz}$ generation (see, e. g. review [9]). It was found that the negative differential conductivity (NDC) causes the formation of electrical domains [10, 11, which makes it difficult to observe the Bloch gain [12]. InAs-based SLs are also highly attractive for studies of these nonlinear phenomena [13], particularly due to the possibility of room temperature operation. Here, we present studies of vertical transport in short-period InAs/AlSb and GaAs/AlAs SLs. The main advantages of these SLs are thin barriers, which ensure a high tunnelling probability, and narrow quantum wells with large energy difference between confined levels, which allows us to take into account only ground states.

\section{Experiment}

The InAs/AlSb SLs grown by molecular beam epitaxy (MBE) consisted of 60 periods of $4.5 \mathrm{~nm}$ InAs/ $3.5 \mathrm{~nm}$ AlSb between the $\mathrm{n}^{+}$top contact layer of InAs: $\mathrm{Si}\left(n=1 \cdot 10^{19} \mathrm{~cm}^{-3}\right)$ and the $\mathrm{n}^{+}$InAs:Si substrate $\left(n=2 \cdot 10^{18} \mathrm{~cm}^{-3}\right)$. InAs quantum wells (QW) were $\mathrm{Si}$ doped with concentration of $5 \cdot 10^{16}$ and $2 \cdot 10^{17} \mathrm{~cm}^{-3}$. The structures have been processed by means of dry (ion beam) etching as well as conventional photolithography to fabricate mesa structures. Then, the plate with the mesas was coated with a dielectric. The last step was a metal (gold) deposition. The ring-shaped metal contacts formed a $\mathrm{THz}$ optical cavity for a freespace wavelength of 110 to $160 \mu \mathrm{m}$ (Fig. 1). (For the ring of $10 \mu \mathrm{m}$ in diameter $D$ shown in Fig. 1, the ringmode wavelength $\lambda=\pi D$ at the refractive index $\sim 3.5$ corresponds just to $110 \mu \mathrm{m}$ in free space).

Practically, it is the microstrip line closed into a ring. The ring resonator can support the excitation of the so-called whispering gallery modes having a sufficiently high quality factor $Q$. For these modes, the 


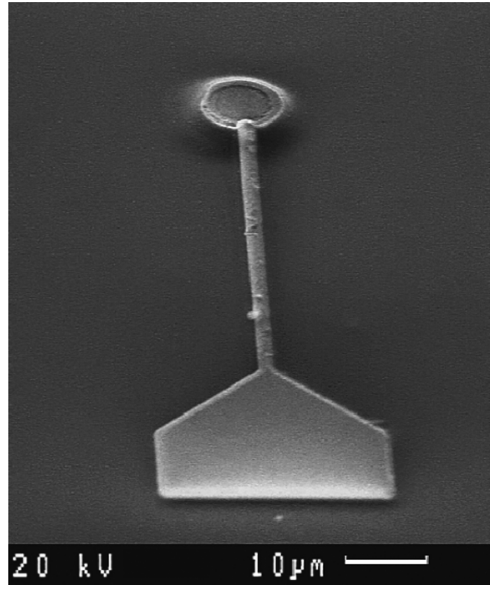

Fig. 1. Resonant cavity with the current lead.

electric field is directed across the SL layers and electromagnetic waves should propagate around the ring.

The current-voltage characteristics were measured both for resonant (with the optical cavity) and nonresonant samples. Rectangular or triangular voltage pulses of 0.2 to $10 \mu$ s duration were applied to the sample. Triangular pulses made it possible to record $I-V$ curves at increasing and decreasing voltages. The measurements were performed at room temperature.

\section{Results and discussion}

Figure 2 shows the current-voltage characteristic of a SL sample without a cavity measured at the matched load resistance. The region with NDC is clearly seen. Naturally, the NDC cannot be measured directly because of electrical domain formation. Only the current jump between the regions with positive differential conductivities, as indicated by the arrow in Fig. 2, can be seen. The NDC is due to the well-known Esaki-Tsu mechanism of miniband transport at the overlapping of broadened confined states in periodic QWs [14].

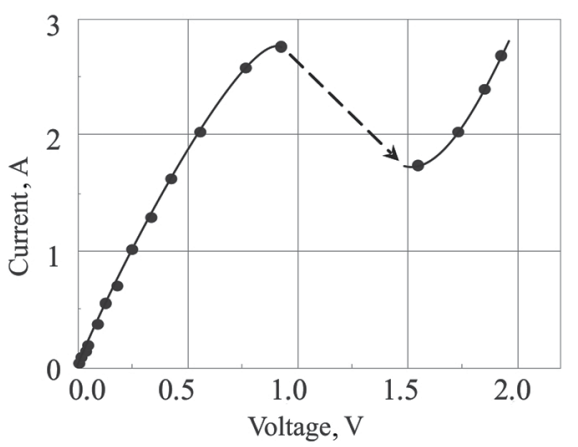

Fig. 2. Current-voltage characteristics of InAs/AlSb SL. Rectangular pulse, matched load resistance, $T=77 \mathrm{~K}$.
At small load resistance, the saturation of the current is observed in the same voltage range (the initial parts of the $I-V$ curves in Figs. 3 and 4 at $U<1.5 \mathrm{~V}$ ). Figure 3 shows that the current saturation is observed for both voltage polarities. The current saturation is due to the static domain formation resulted from the NDC by the Esaki-Tsu mechanism.

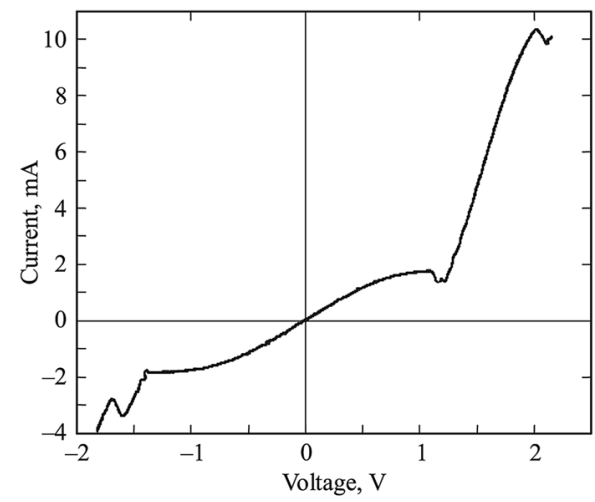

Fig. 3. Current-voltage characteristics of InAs/AlSb SL in two polarities. Triangular pulse, small load resistance, $300 \mathrm{~K}$.

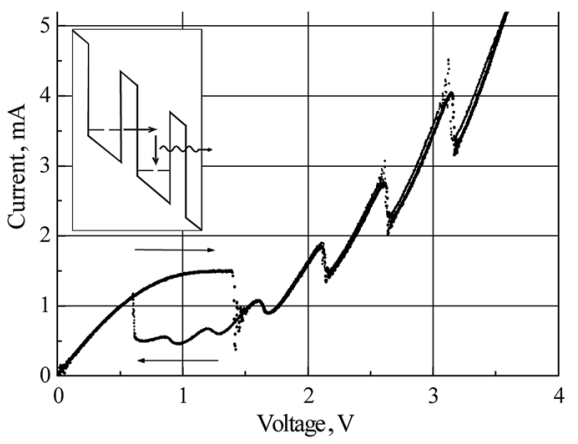

Fig. 4. Current-voltage characteristics of resonant InAs/ AlSb SL structure. $D=14 \mu \mathrm{m}$. Triangular pulses, small load resistance. Inset: Scheme of non-resonant tunnelling.

The estimated miniband width for our InAs/AlSb SLs is about $26 \mathrm{meV}$. The ends of the saturation regions in Figs. 3 and 4 for the SL of 60 periods correspond to $\sim 1.4 \mathrm{~V} / 60=23 \mathrm{mV}$ per SL period. At higher voltages, the overlapping of the ground confined states in the neighbouring QWs should disappear and the conductivity will be governed by non-resonant tunnelling between QWs assisted with energy relaxation through acoustic phonon emission. This mechanism should lead to the monotonic rise of the current (see, e. g. [9]).

However, several equidistant maxima (up to 6) are observed in the voltage range of $1.5-4.5 \mathrm{~V}$ in the 
$I-V$ characteristics (Fig. A). For the given sample, the distances between these maxima are $\sim 8.3 \mathrm{mV}$ per SL period. They cannot originate from the overlapping of the ground confined state in a QW with upper excited states in a neighbouring QW because the energy difference between these states is, in our case (narrow QW and small effective mass), larger than $0.4 \mathrm{eV}$ (the calculated energies and wave functions for two lower confined levels are presented in Fig. 5). Such overlapping can be reached only at a voltage by one order of magnitude larger than that used in our experiments.

Note that distinctive features were observed in 115 in $I-V$ characteristics of GaAs/AlGaAs SLs with thin barriers and wide enough QWs (at liquid He temperature), which were attributed to resonant (inter-miniband) tunnelling between the QWs located at several SL periods from each other. In contrast to that work, we have to admit for our SLs that, first, the possibility of electron tunnelling across 10-13 SL periods would be possible and, second, the maxima in $I-V$ curves would not be equidistant.

The maxima observed cannot either be attributed to non-resonant tunnelling assisted with the emission of the optical phonon because its energy in InAs is $\sim 30 \mathrm{meV}$.

We attribute the periodic maxima in the $I-V$ characteristics to the influence of the resonant cavity on spontaneous electromagnetic emission due to the Purcell effect [16]. In this case, the intensity of spontaneous emission at the resonant frequency has to be much larger than that without the cavity. The enhancement of a spontaneous emission rate can be estimated using the Purcell factor

$$
F_{\mathrm{P}}=\left(3 / 4 \pi^{2}\right)\left(\lambda^{3} / V\right) Q
$$

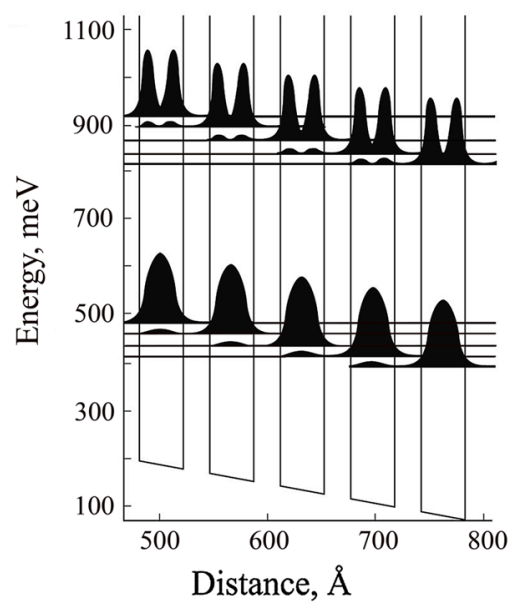

Fig. 5. Energies and wave functions for InAs/AlSb SLs, $40 \mathrm{kV} / \mathrm{cm}, T=300 \mathrm{~K}$. where $\lambda$ is the wavelength in the material, $Q$ is the quality factor of the cavity, and $V$ is the mode volume. Assuming $Q=100$, we get $F_{\mathrm{p}}$ about $10^{3}$. Really, the $Q$ factor for whispering gallery modes in ring microcavities can be orders of magnitude larger: $\sim 510^{5}$ [17]. This means that the energy loss because of the emission of a resonant-frequency photon at non-resonant tunnelling may become comparable with that at the emission of acoustic phonons. As a result, an additional increase in the current should be observed near the resonant frequencies. In the opposite case, when the resonant photon energy is far from the energy of electron transition between the confined levels in the neighbouring QWs, the radiative transitions should be strongly suppressed and the current will come down to the value at the acoustical phonon emission. The maxima in the current-voltage characteristic will appear at the voltages corresponding to the energies of neighbouring resonances of the cavity. In a ring cavity, the resonant frequencies correspond to an integer number of wavelengths at the ring perimeter, and the difference between the photon energies in neighbouring resonances must coincide with the photon energy of the main mode with $\lambda \approx \pi D$ ( $D$ is the ring diameter). The sample, for which the results are presented in Fig. 4 , has the ring diameter of $14 \mu \mathrm{m}$ and the observed voltage difference between the peaks is well correlated with the energy of the photon with the frequency of our cavity $(\sim 8 \mathrm{meV})$.

Similar effect was observed in short-period GaAs/ AlAs SLs with the ring resonator. The current-voltage characteristic is presented in Fig. 6. Many maxima are observed in these SLs, but the behaviour of the current is more complicated than in InAs/AlSb SLs. First, the usual region of the miniband transport is practically absent. It is likely due to smaller barrier widths and heights, what makes non-resonant tunnelling much more effective. Second, at least two periods of the current peaks can be seen. A smaller one likely corresponds to the tunnelling of electrons across two

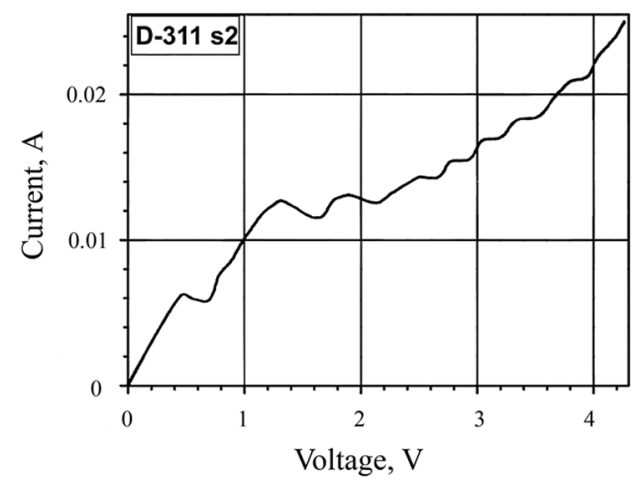

Fig. 6. Current-voltage characteristics of GaAs/AlAs SL, $T=300 \mathrm{~K}$. Triangular pulses, $1 \mathrm{Ohm}$ load resistance. 
successive barriers. The periods agree rather well with the resonant energy of the ring cavity; it is close to $\sim 8 \mathrm{meV}$.

\section{Concluding remarks}

The periodic maxima observed in the current-voltage characteristics of short-period superlattices were attributed to the Purcell effect. Certainly, additional confirmation is desirable for this explanation, both theoretical and experimental. In particular, it is necessary to calculate the probabilities of phonon-assisted and radiative transitions between the confined states in the neighbouring QWs. It would be useful as well to study the current-voltage characteristics of resonant cavity samples with different frequencies.

\section{Acknowledgements}

The work is supported by RFBR grants 11-02-00611 and 12-02-31828, RAS programs "Basic research in nanotechnology and nanomaterials" and "Modern problems of radio physics", and the Ministry of Science and Education (Agreement 8514).

\section{References}

[1] J. Hoffmann, T. Lehnert, D. Hoffmann, and H. Fouckhardt, Semicond. Sci. Technol. 24, 065008 (2009).

[2] J. Guo, Z. Peng, W. Sun, Y. Xu, Z. Zhou, and Z. Niu, Infrared Phys. Technol. 52, 124 (2009).

[3] J.B. Rodriguez, E. Plis, G. Bishop, Y.D. Sharma, H.S. Kim, L.R. Dawson, and S. Krishna, Appl. Phys. Lett. 91, 043514 (2007).
[4] L.L. Li, W. Xu, Z. Zeng, A.R. Wright, C. Zhang, J. Zhang, Y.L. Shi, and T.C. Lu, Microelectronics J. 40, 812 (2009).

[5] A.N. Baranov, N. Bertru, Y. Cuminal, G. Boissier, C. Alibert, and A. Joullié, Appl. Phys. Lett. 71, 735 (1997).

[6] A.A. Popov, V.V. Sherstnev, A.N. Baranov, C. Alibert, and Y.P. Yakovlev, Electronics Lett. 34, 1398 (1998).

[7] J. Devenson, R. Teissier, O. Cathabard, and A.N. Baranov, Appl. Phys. Lett. 90, 111118 (2007).

[8] T. Daoud, G. Boissier, J. Devenson, A.N. Baranov, and R. Teissier, J. Phys. Conf. Series 193, 012014 (2009).

[9] A. Wacker, Phys. Rep. 357, 1-111 (2002).

[10] M. Büttiker and H. Thomas, Phys. Rev. Lett. 38, 78 (1977).

[11] A.A. Ignatov and V.I. Shashkin, Zh. Eksp. Teor. Fiz. 93, 935 (1987) [Sov. Phys. JETP 66, 526 (1987)].

[12]F. Klappenberger, K.N. Alekseev, K.F. Renk, R. Scheuerer, E. Schomburg, S.J. Allen, G.R. Ramian, J.S.S. Scott, A. Kovsh, V. Ustinov, and A. Zhukov, Eur. Phys. J. B 39, 483 (2004).

[13] M.S. Kagan, I.V. Altukhov, A.N. Baranov, N.D. Il'inskaya, S.K. Paprotskiy, V.P. Sinis, and A.A. Usikova, Acta Phys. Polonica. A 119, 210 (2011).

[14] L. Esaki and R. Tsu, IBM J. Res. Develop. 14, 61 (1970).

[15] A.A. Andronov, E.P. Dodin, D.I. Zinchenko, and Yu.N. Nozdrin, Semiconductors 43, 228 (2009) [Fiz. Tekh. Poluprovodn. 43, 240 (2009)].

[16] E.M. Purcell, Phys. Rev. 69, 681 (1946).

[17] L. Tobing and P. Dumon, Fundamental principles of operation and notes on fabrication of photonic microresonators, in: Photonic Microresonator Research and Applications, Springer series in Optical Sciences, Vol. 156, (2010), p. 1-27.

\title{
NEREZONANSINIS TUNELIAVIMAS TRUMPO PERIODO SUPERGARDELĖSE SU OPTINIAIS REZONATORIAIS
}

\author{
M.S. Kagan ${ }^{\text {a }}$, I.V. Altukhov a, S.K. Paprotskiy a, A.N. Baranov ${ }^{\text {b }}$, R. Teissier ${ }^{\text {b }}$, N.D. Il'inskaya ${ }^{\text {c }}$, \\ A.A. Usikova ${ }^{c}$, A.D. Buravlev ${ }^{c}$, V.M. Ustinov ${ }^{c}$ \\ ${ }^{\text {a }}$ Rusijos mokslu akademijos Kotelnikovo Radijo ir elektronikos inžinerijos institutas, Maskva, Rusija \\ ${ }^{\mathrm{b}}$ Monpelje II universitetas, Monpelje, Prancūzija \\ 'Rusijos mokslu akademijos Jofés fizikos technikos institutas, Sankt Peterburgas, Rusija
}

